\title{
Spectroscopic properties and dynamical evolution of the merging system AM 1003-435 ^
}

\author{
G. I. Günthardt ${ }^{1,2, \star \star}$, E. L. Agüero ${ }^{1}$, I. Rodrigues ${ }^{5}$, and R. J. Díaz ${ }^{1,3,4}$ \\ 1 Observatorio Astronómico, Universidad Nacional de Córdoba, Laprida 854, 5000 Córdoba, Argentina \\ e-mail: [gunth; aguero; diaz]@mail.oac.uncor .edu \\ 2 SECyT, Universidad Nacional de Córdoba, Argentina \\ 3 CONICET, Argentina \\ ${ }^{4}$ Gemini Observatory, Southern Operation Center, c/o AURA, La Serena, Chile \\ 5 Instituto de Fisica, Universidade Federal do Rio Grande do Sul, CEP 91501-970, Porto Alegre, RS, Brazil \\ e-mail: irapuan@if.ufrgs.br
}

Received 29 June 2004 / Accepted 2 December 2005

ABSTRACT

\begin{abstract}
Aims. We study the system AM 1003-435, which is composed of two strong interacting galaxies.
Methods. We obtained long-slit optical spectra of twelve zones of the system, and performed numerical simulations of the encounter between the components following the evolution of their stellar and gaseous contents.

Results. The spectrum of the NW nucleus is typical of a starburst, while that of the SE one shows weak emission lines. The highest values of the oxygen and nitrogen abundances are at the NW nucleus. Its derived $\mathrm{H} \alpha$ and $\mathrm{H} \alpha+[\mathrm{N} \mathrm{II}]$ equivalent widths indicate very intense star formation, in accord to its starburst nature. Indicative ages of the starbursts in the nuclei were obtained. The morphological types of both components derived from their spectral characteristics are in agreement with previous determinations based on photometric parameters. About $70 \%$ of the measured $\mathrm{H} \alpha$ luminosity would correspond to the NW component contribution. The IR luminosity of the system is not high $\left(L_{\mathrm{IR}}<10^{11} L_{\odot}\right)$. The estimated star formation rate for AM 1003-435 indicates that its activity is also moderate. The IR radiation, if it has the same origin as the $\mathrm{H} \alpha$ emission, would arise mostly from the NW component. The resulting abundances, burst ages, and masses suggest that the starburst in the SE component, the minor one, started earlier than that of the NW one, and did so in a medium poorer in weighted elements. On the other hand, from a set of $N$-body simulations of the encounter between both components it was found the time of the perigalacticum, to be comparable to the burst age derived for the SE component, and the star formation in both galaxies would have begun after the perigalacticum. It was also estimated that the central bodies will merge in about $1 \mathrm{Gyr}$.
\end{abstract}

Key words. galaxies: individual: AM 1003-435 - galaxies: interactions - galaxies: nuclei - techniques: spectroscopic methods: $N$-body simulations

\section{Introduction}

In the last fifty years there has been continuous growth in the observational and theoretical scenario of the links between interactions, star formation, and galaxy evolution. Notwithstanding, there is a lack of precision in the exact nature of the links between different types of interactions and different types of galaxies, and more detailed anatomic studies of individual objects are required. We are developing a systematic study of objects that although, well suited for testing the present physical knowledge of galaxy interactions, have been poorly reviewed in the literature.

In the present case of the system AM 1003-435 (ESO 263IG003, IRAS 10037-4358, SGC 1003-440) the most remarkable morphological features are two components, or nuclei separated by about $36^{\prime \prime}$ (equivalent to $\sim 7.8 \mathrm{kpc}$, considering $H_{0}=$ $75 \mathrm{~km} \mathrm{~s}^{-1} \mathrm{Mpc}^{-1}$ ) connected by an apparent bridge that contains some bright condensations. All of this is within a diffuse

* Table 1 is only available in electronic form at http://www .edpsciences.org

$\star \star$ Visiting astronomer at the Complejo Astronómico El Leoncito (CASLEO), which is operated under agreement between the Consejo Nacional de Investigaciones Científicas y Técnicas de la República Argentina and the National Universities of La Plata, Córdoba, and San Juan. envelope that extends in two long opposed tails giving to the system the aspect of a distorted "S" (Fig. 1), indicative of strong gravitational interactions between components or of a merging process. On I and IR direct images (from DSS images, for instance) the central regions of the SE and NW components present spherical and elliptical shapes, respectively; very close to this last one there are two stars that, on some blue images, give it a tri-lobed appearance. Although the system seems to be in a strongly interacting phase of two disk galaxies, its infrared luminosity $\left(L_{\mathrm{IR}}<10^{11} L_{\odot}\right)$ is moderate, and the question arises as to what role has been played both by the structure of the progenitor galaxies and by the relative orbits in the star formation history of this particular system.

\section{Observations and reductions}

The spectroscopic observations of AM 1003-435 were carried out on two epochs. The first one was carried out on 2 April 2003 with a REOSC spectrograph coupled to the $2.15 \mathrm{~m}$ Ritchey-Chrétien telescope of the Complejo Astronómico El Leoncito (CASLEO), San Juan, Argentina. The detector was a Tektronix CCD $1024 \times 102424 \mu$ m pixels. The seeing during observations was $2^{\prime \prime}-3^{\prime \prime}(F W H M)$. Three exposures of 20 min each were obtained through a slit of $2.8^{\prime \prime}$ wide 

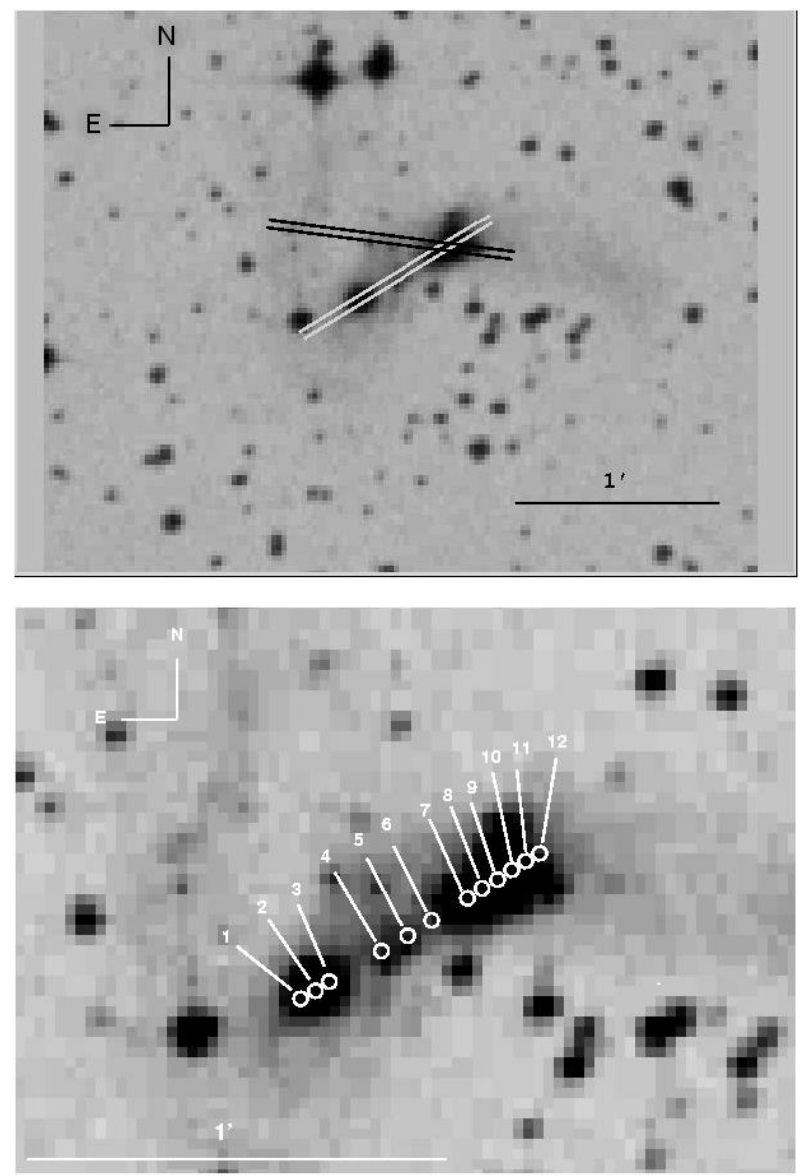

and $348^{\prime \prime}$ long, along the direction that joins both nuclei $(\mathrm{PA}=$ $123^{\circ}$; Fig. 1), using a 300 -line $\mathrm{mm}^{-1}$ grating covering the wavelength range 4200-7000 $\mathrm{A}$. The dispersion was $129 \AA \mathrm{mm}^{-1}$, the reciprocal dispersion $3.1 \AA$ per pixel, and the resolution $8 \AA$. The spectra were corrected for atmospheric and Galactic extinction, and the flux was calibrated with stars from the cata$\log$ of Stone \& Baldwin (1982). The angular scale was 1.02" pixel $^{-1}$. On 10 March 2005, observations were performed using the same instrumental configuration mentioned above, but with a 1200 -line $\mathrm{mm}^{-1}$ grating, covering a wavelength range of 6300-7000 $\AA$. The dispersion was $44 \AA \mathrm{mm}^{-1}$, the reciprocal dispersion $0.62 \AA$ per pixel, and the resolution $3 \AA$. Three exposures of $60 \mathrm{~min}$ each were taken through a slit of $3.3^{\prime \prime}$ wide and $348^{\prime \prime}$ long, with a position angle of $78^{\circ}$, which covers the centre of the NW component and intersects the northern arm. Data reduction of the spectra was made employing the standard methods of the IRAF (developed by NOAO) reduction package.

\section{Spectroscopy}

Spectroscopic information from the centres of the two nuclei, their neighbouring zones, and three emission regions of the bridge mentioned above were all obtained (Fig. 1 and Table 1). The spectra of the NW nuclear region (Fig. 2a) present strong emission lines typical of starbursts, while those of the SE one (Fig. 2b) show weak emission lines. In order to analyse the isolated line emission, selected templates from Bica (1988) were subtracted whenever possible, to discount the spectral stellar population contribution. It was found that the S7 and S2 templates were the best representations of the stellar populations in the NW and SE nuclei, respectively. Thus the dominant stellar
Fig. 1. Top: slit width superposed on a blue image (plate from the "Digitized Sky Survey") of the system AM 1003-435; bottom: the twelve regions of AM 1003-435 from which spectroscopic information was derived.

population in the NW nuclear region would be of A type, while in the SE nuclear region a $\mathrm{K}$ stellar type predominate. Those spectra were influenced by the evolved stars in the progenitor bulges, which explains the fitted stellar populations. The three regions associated to the bridge also present emission lines and very weak continua.

For the selected twelve regions the measured and corrected line intensities $F_{\lambda}$ and $I_{\lambda}$ relative to $\mathrm{H} \beta=1.00$ (regions 2 and 9 correspond respectively to the centres of the SE and NW nuclear regions) are listed in Table 1, along with the errors, estimated from the noise level around each line. The values of $c$ and the corrected $\mathrm{H} \beta$ fluxes are given at the bottom of this table. In a classical diagnostic diagram from Veilleux \& Osterbrock (1987), $\log ([\mathrm{O} \mathrm{III}] \lambda 5007 / \mathrm{H} \beta)$ vs. $\log ([\mathrm{N} \mathrm{II}] \lambda 6583 / \mathrm{H} \alpha)$, the ratios corresponding to the studied regions are plotted. All the measured values lie in the H II region emission zone (see Fig. 3).

\subsection{Relative oxygen and nitrogen abundances, electron densities, and temperatures}

The relative abundances of oxygen $N(\mathrm{O}) / N(\mathrm{H})$ and nitrogen $N(\mathrm{~N}) / N(\mathrm{H})$, and the electron temperatures $T_{\mathrm{e}}$ and densities $N_{\mathrm{e}}$ were also calculated. The average values from the empirical calibration of Edmunds \& Pagel (1984) were adopted for the $N(\mathrm{O}) / N(\mathrm{H})$ abundances. The $N(\mathrm{~N}) / N(\mathrm{H})$ abundances were derived by making the usual assumptions valid for $\mathrm{H}$ II regions. Since the [O II] $\lambda 3727$ lines are not within our wavelength coverage, the necessary $\lambda 3727 / \mathrm{H} \beta$ ratios were derived using the first predicting equation given by McCall et al. (1985). The electron temperatures were obtained by searching the required values of $T_{\mathrm{e}}$ for the adopted $N(\mathrm{O}) / N(\mathrm{H})$ abundances. The electron 


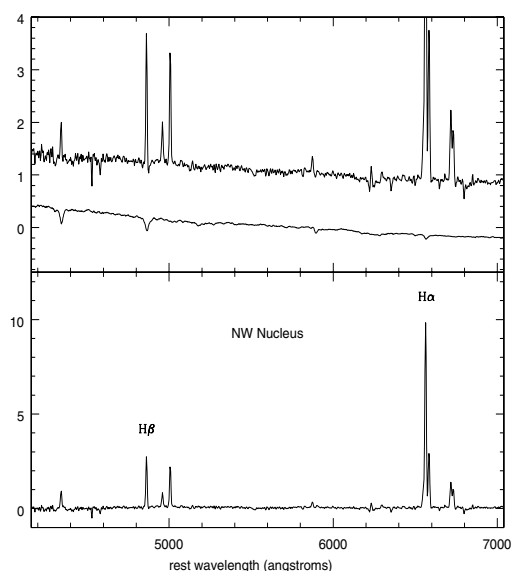

(a)

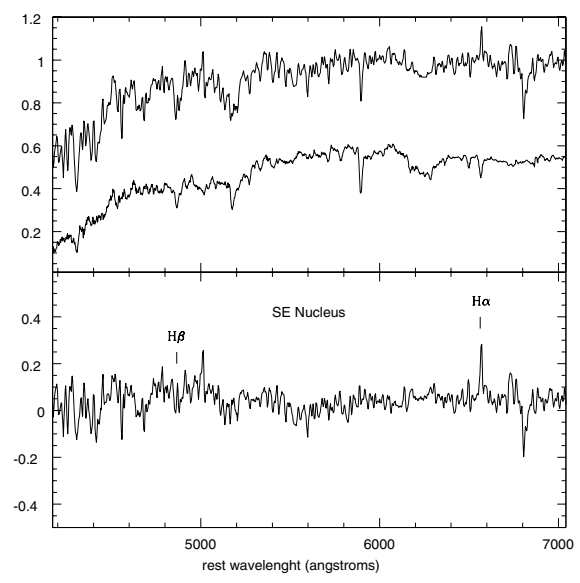

(b)
Fig. 2. a) (Upper panel) Spectrum of the NW nucleus of AM 1003-435 before (top) the subtraction of the stellar population template S7 (bottom). (Lower panel) Spectrum of the NW nucleus after the subtraction of this template. Ordinate in arbitrary units. b) (Upper panel) Spectrum of the SE nucleus of AM 1003-435 before (top) the subtraction of the stellar population template S2 (bottom). (Lower panel) Spectrum of the SE nucleus after the subtraction of this template. Ordinate in the same arbitrary units as in plot a).

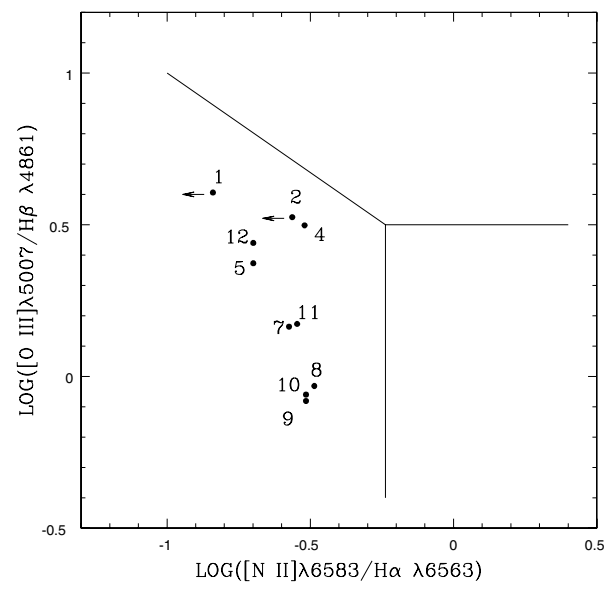

Fig. 3. $\operatorname{LOG}([\mathrm{O} I I I] \lambda 5007 / \mathrm{H} \beta)$ vs. $\operatorname{LOG}([\mathrm{N} \mathrm{II}] \lambda 6583 / \mathrm{H} \alpha)$ for the studied regions. Considering that it not was possible to obtain reliable measures of $F_{[\mathrm{NII}] \lambda 6583}$ in regions 1 and 2 , due to the continuum noise, and considering this noise as a limit for the flux of the nitrogen line, we estimate that in this case $F_{[\mathrm{NII}] \lambda 6583} / \mathrm{F}_{\beta}$ would not be greater than about 1 , so this fixes a maximun value for $F_{[\mathrm{NII}] \lambda 6583} / F_{\mathrm{H} \alpha}$.

densities $N_{\mathrm{e}}$ were derived from the [S II] $\lambda 6717 / \lambda 6731$ ratios (Osterbrock 1989). These densities, although quite low, are in the range of normal values for H II regions, as well as the derived electron temperatures. These results are listed in Table 2. The relative oxygen and nitrogen abundances of the NW nucleus (region 9) are comparable to those of galactic emission regions ( $\sim 1$ and 1.2 of them respectively), and $\sim 0.8$ and 0.4 of the solar ones; its ratio $\mathrm{N} / \mathrm{O}=0.06$ is coincident with the normal values in galactic regions, being about the half of the respective solar ratio, which reflects a comparative overabundance of $\mathrm{O}$ with respect to N. In the SE nucleus (region 2) the relative oxygen abundance is $\sim 0.4$ of those of the galactic emission regions and $\sim 0.3$ of the corresponding solar abundance. The distributions of these abundances (Figs. 4a and b) present their highest values at the NW nucleus and decrease with the distance from it, as has been observed in the disks of many spiral galaxies (Evans 1986; Shields 1990). The zones of the apparent bridge continue the decreasing trend mentioned above. In the SE component, the values of the oxygen abundance are comparatively low.

\subsection{Equivalent widths}

The equivalent widths $E W(\mathrm{H} \alpha)$ (Table 2 and Fig. 4c) and $E W(\mathrm{H} \alpha+[\mathrm{N} \mathrm{II}])$ of the selected regions, of the integrated spectra of the nuclear regions, and of the integrated spectrum of the system were measured. For the NW nucleus (region 9) they resulted in $E W(\mathrm{H} \alpha)=119 \AA$ and $E W(\mathrm{H} \alpha+[\mathrm{N} \mathrm{III}])=173 \AA$, values indicative of very intense star-formation activity that confirms its starburst nature (Liu \& Kennicutt 1995), while for the SE nucleus (region 2) $E W(\mathrm{H} \alpha)=2 \AA$ was found. Indicative ages of $\sim 6.2 \times 10^{6} \mathrm{yr}$ and $\sim 2.2 \times 10^{7} \mathrm{yr}$ were obtained for their bursts of star formation according to the model for instantaneous bursts with metallicities of $0.6 Z_{\odot}$ and $0.3 Z_{\odot}$ (values derived from the similar curves corresponding to 1 and $0.2 Z_{\odot}$, and to 0.2 and $0.4 Z_{\odot}$ from Leitherer et al. 1999).

The integrated spectrum of the NW component, typical of a starburst (Liu \& Kennicutt 1995), with $E W(\mathrm{H} \alpha)=87 \AA$ and $E W(\mathrm{H} \alpha+[\mathrm{N} \mathrm{II}])=126 \AA$, corresponds to a galaxy of $\mathrm{Sc} / \mathrm{Sm}$ type. It is also very similar to that of NGC 4194, an $\mathrm{Sm} / \mathrm{Im}$ pec galaxy that is also dominated by a starburst and that shows evidence of a recent merger (Kennicutt 1992). The integrated spectrum of the SE component, characteristic of slightly active star formation systems (Liu \& Kennicutt 1995), corresponds to Sa-Sab type galaxies. In these morphological types the $\mathrm{H} \alpha$ equivalent width is usually only of a few angstroms, in agreement with the one found here $E W(\mathrm{H} \alpha) \sim 4 \AA$. We derived $E W(\mathrm{H} \alpha)=46 \AA$ and $E W(\mathrm{H} \alpha+[\mathrm{N} \mathrm{II}])=62 \AA$ from the integrated spectrum of the system AM 1003-435. For mergers it is found on average $E W(\mathrm{H} \alpha+[\mathrm{N} I \mathrm{II}])=59 \AA$ indicating significantly enhanced star-formation rates in comparison to isolated galaxies (Liu \& Kennicutt 1995), thus the corresponding value of $62 \AA$ obtained here reflects the merger nature of the system as its morphology suggests. On the other hand, the morphological types assigned here to both components according to their spectral characteristics agree with the Lauberts \& Valentijn (1989) classification based on photometric parameters.

The very different values for the equivalent widths derived from the three mentioned integrated spectra would indicate that there is no global star-formation activity, and that this one is particularly intensified in the NW nuclear region. Figure $4 \mathrm{c}$ also shows that the starburst activity is appreciable in the apparent bridge and comparatively low in the SE nuclear region. As a result, the observed star-formation activity, presumably induced by interactions, does not take place in both nuclei. This is compatible with the enhanced activity detected by Joseph et al. (1984) and Bernlöhr (1993) in only one nucleus of pairs of galaxies, 
Table 2. Physical parameters.

\begin{tabular}{|c|c|c|c|c|c|c|c|c|c|c|c|c|}
\hline Parameter & $\begin{array}{c}\text { Reg. } 1 \\
40.9\end{array}$ & $\begin{array}{c}\text { Reg. } 2 \\
36 . " 4 \\
\end{array}$ & $\begin{array}{c}\text { Reg. } 3 \\
344^{\prime \prime} 8 \\
\end{array}$ & $\begin{array}{l}\text { Reg. } 4 \\
24 . " 4 \\
\end{array}$ & $\begin{array}{c}\text { Reg. } 5 \\
16 . " 8 \\
\end{array}$ & $\begin{array}{c}\text { Reg. } 6 \\
10.7 \\
\end{array}$ & $\begin{array}{c}\text { Reg. } 7 \\
6{ }^{\prime \prime} 1 \\
\end{array}$ & $\begin{array}{c}\text { Reg. } 8 \\
\text { 3'. } 1 \\
\end{array}$ & $\begin{array}{c}\text { Reg. } 9 \\
0^{\prime \prime}\end{array}$ & $\begin{array}{c}\text { Reg. } 10 \\
-3{ }^{\prime \prime} 1 \\
\end{array}$ & $\begin{array}{c}\text { Reg. } 11 \\
-6{ }^{\prime \prime} 1 \\
\end{array}$ & $\begin{array}{c}\text { Reg. } 12 \\
-9 . ' 1 \\
\end{array}$ \\
\hline$\frac{N(\mathrm{O})}{N(\mathrm{H})} \times 10^{4}$ & $2.0 \pm 1.8$ & $2.2 \pm 2.0$ & - & $2.4 \pm 0.5$ & $3.1 \pm 1.0$ & - & $3.8 \pm 1.5$ & $5.1 \pm 1.0$ & $5.6 \pm 0.6$ & $5.6 \pm 0.3$ & $4.3 \pm 0.4$ & $2.8 \pm 1.3$ \\
\hline$\frac{N(\mathrm{~N})}{N(\mathrm{H})} \times 10^{5}$ & - & - & - & $1.4 \pm 1.4$ & $1.6 \pm 1.6$ & - & $1.9 \pm 1$ & $3.6 \pm 1.8$ & $3.6 \pm 1.7$ & $3.6 \pm 1.7$ & $2.9 \pm 1.6$ & $1.9 \pm 1.7$ \\
\hline$\frac{N(\mathrm{~N})}{N(\mathrm{O})}$ & - & - & - & 0.06 & 0.05 & - & 0.05 & 0.07 & 0.06 & 0.06 & 0.07 & 0.07 \\
\hline$T_{\mathrm{e}}(\mathrm{K})$ & 10100 & 9850 & - & 9180 & 8780 & - & 8185 & 7540 & 7370 & 7390 & 8000 & 9080 \\
\hline$N_{\mathrm{e}}\left(\frac{1}{\mathrm{~cm}}\right)^{3}$ & - & - & - & - & - & - & - & 10 & 10 & 10 & 10 & - \\
\hline$E W(\mathrm{H} \alpha)(\AA)$ & $2 \pm 0.25$ & $2 \pm 0.25$ & $2 \pm 0.25$ & $71 \pm 37$ & $79 \pm 28$ & $56 \pm 12$ & $32 \pm 11$ & $90 \pm 7$ & $119 \pm 8$ & $92 \pm 10$ & $53 \pm 7$ & $39 \pm 17$ \\
\hline$V_{u}\left(\mathrm{~km} \mathrm{~s}^{-1}\right)$ & $3350+18$ & $3341 \pm 28$ & $3308 \pm 16$ & $3236 \pm 40$ & $3301 \pm 34$ & $3368 \pm 40$ & $3357 \pm 24$ & $3362+28$ & $3386+10$ & $3380+1$ & $3316+1$ & $3274+36$ \\
\hline
\end{tabular}

Note: Distances are as in Table 1.

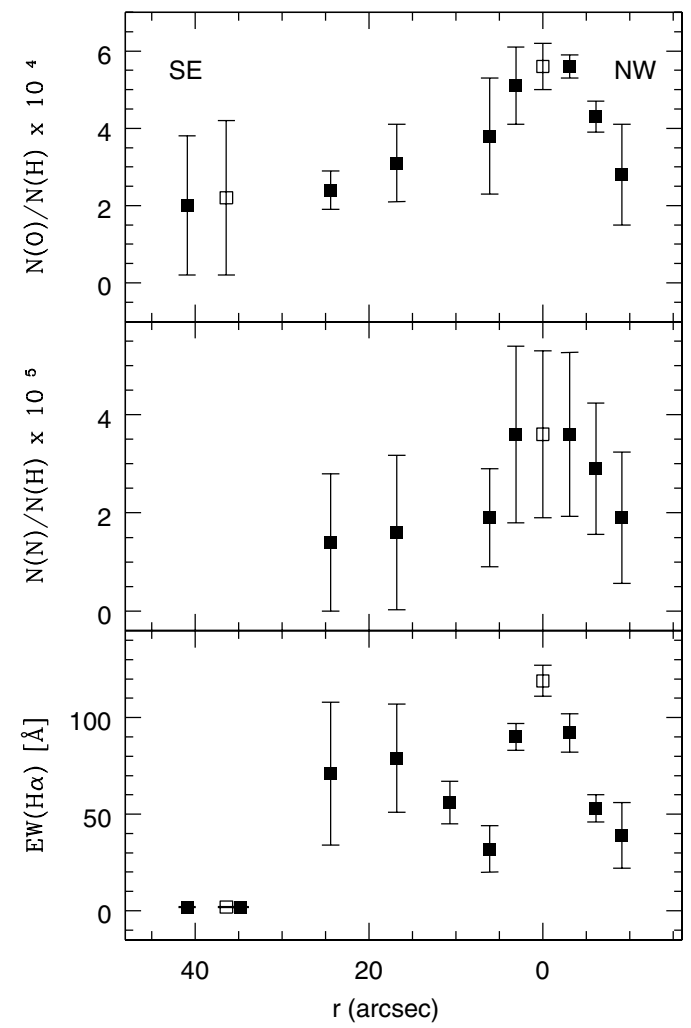

Fig. 4. a) Abundance ratio $N(\mathrm{O}) / N(\mathrm{H})$ vs. distance. Open squares correspond to the centre of the SE (region 2) and NW (region 9) nuclear regions. Distances are given with respect to region 9 , and are positive towards the Southeast. b) Abundance ratio $N(\mathrm{~N}) / N(\mathrm{H})$ vs. distance. c) $\mathrm{H} \alpha$ equivalent width vs. distance. The errors of the oxygen abundances were estimated taking into account the errors of the line fluxes that were used in the Edmunds \& Pagel (1984) plots. The errors of the nitrogen abundances were estimated by propagating errors in the ionic abundances expressions of Díaz (1985).

and by Agüero et al. (2000) in another object with two nuclei; on the other hand, it differs from the results of Sekiguchi \& Wolstencroft (1992) who observed that same activity in both nuclei of some pairs of galaxies. The low star-formation in the SE nucleus, in spite of the interactions supposed by present, suggests that AM 1003-435 is seen after the peak of activity of the SE progenitor. This one would have little material available for star-formation, while the NW component would have enough gas to sustain the observed starburst. This is compatible with the morphological types of these components derived here from their integrated spectra.

\subsection{Luminosities}

The $\mathrm{H} \alpha$ luminosities derived from the integrated spectra of the AM 1003-435 system and the NW component, which include most of the $\mathrm{H} \alpha$ emission, are $\left(L_{\mathrm{H}_{\alpha}}\right)_{\mathrm{S}} \geq 3 \times 10^{7} L_{\odot}$ and $\left(L_{\mathrm{H}_{\alpha}}\right)_{\mathrm{NW}} \geq 2 \times 10^{7} L_{\odot}$ respectively, so that about $70 \%$ of the $\left(L_{\mathrm{H}_{\alpha}}\right)_{\mathrm{S}}$ would correspond to the NW component contribution. These values are lower limits because there should be some $\mathrm{H} \alpha$ contribution originated outside of the slit coverage, which comprises 610 pc. Nevertheless, in the best-studied case of M 82 the starburst and, therefore, its $\mathrm{H} \alpha$ luminosity are confined within a region of $\sim 500 \mathrm{pc}$ in size (Moorwood 1996). Moreover, if the starburst is extended to the blue regions in the colour map by Reduzzi \& Rampazzo (1996), then a minimun of $50 \%$ of the $\mathrm{H} \alpha$ emission should have been covered by the slit, when also considering the peakness of the profile in Fig. 5. Therefore, we estimate a maximun $\mathrm{H} \alpha$ luminosity $\left(L_{\alpha}\right)_{\mathrm{NW}} \leq 10^{8} L_{\odot}$.

Gravitational interactions would not have intensified the IR emission of AM 1003-435 significantly in relation to other mergers, because $L_{\mathrm{IR}}=2.5 \times 10^{10} L_{\odot}$. This luminosity was obtained from IRAS data, and by adopting FIR from Sanders \& Mirabel (1996). This value is comparable to those of the archetypicals M 82, NGC 253, and NGC 7714, starburst galaxies of moderate activity which have infrared luminosities of a few $10^{10} L_{\odot}$. The low spatial resolution of the IRAS data makes the infrared flux source uncertain but, if it is accepted that the IR radiation has the same origin as the $\mathrm{H} \alpha$ emission, we can consider that the IR radiation arises mostly from the NW component. The far infrared colour indexes $\alpha(60,25)$ and $\alpha(100,60)$ also point AM 1003-435 as a starburst, in agreement with the spectroscopic data.

An estimate of the star-formation rate was derived through $\mathrm{d} M / \mathrm{d} t=7 \times 10^{11}\left(L_{\mathrm{IR}} / L_{\odot}\right)\left[M_{\odot} / \mathrm{yr}\right]($ Scoville \& Young 1983) $\sim 2\left[M_{\odot} / \mathrm{yr}\right]$, which is very close to those of M 82 and NGC 253 and which shows that the corresponding activity in AM 1003-435 is moderate, too.

\subsection{Radial velocities}

Radial velocities of the studied zones were derived (see Table 2) by measuring the centroids of Gaussians curves fitted at the strongest emission lines. We checked that the errors of the fitting provided by the IRAF task rvidlines (multiplied by two) were the same order of magnitude as the differences in 


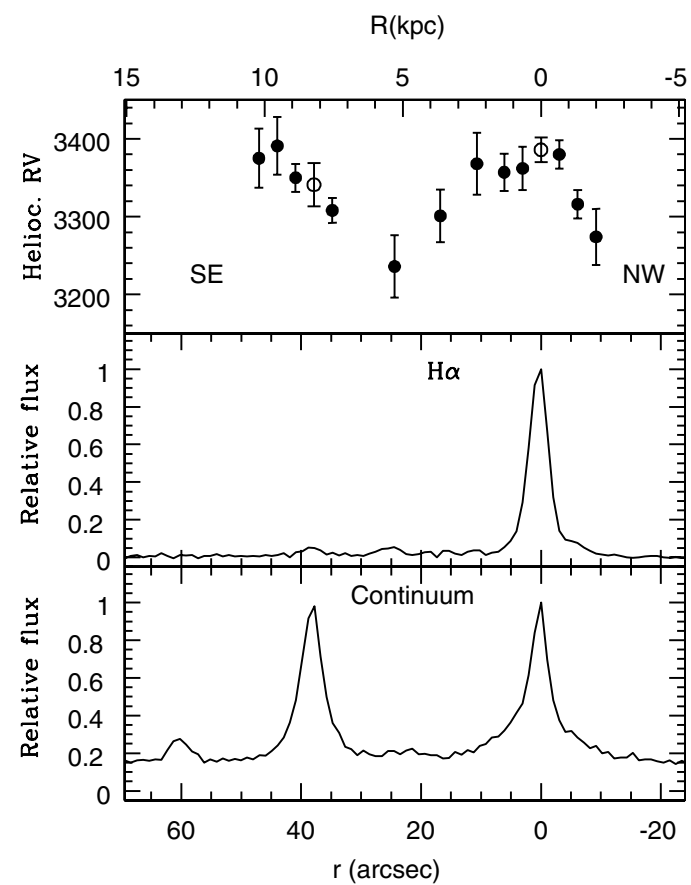

Fig. 5. (Top) Heliocentric radial velocity distribution along $\mathrm{PA}=123^{\circ}$. The values correspond to the weighted average velocities of the brightest emission lines. Open circles correspond to the centre of the SE (region 2) and NW (region 9) nuclear regions. Distances are as in Fig. 4. (Middle) $\mathrm{H} \alpha$ flux distribution (continuum subtracted) along the same position angle. (Bottom) Continuum distribution along the same position angle.

velocity that result from adding portions of the adjacent continuum, in order to evaluate the differences produced by the fitting noise. For this reason, we adopted the criteria of reporting $2 \sigma$ as the uncertainty, where $\sigma$ is the error given by the IRAF task.

The resulting heliocentric radial velocities of the NW and SE nuclei are $V_{\mathrm{NW}}=(3386 \pm 20) \mathrm{km} \mathrm{s}^{-1}$ and $V_{\mathrm{SE}}=(3341 \pm$ $30) \mathrm{km} \mathrm{s}^{-1}$ respectively. The average velocity was adopted as the systemic velocity of AM 1003-435, which refers to the Galactic system of rest as $V_{\mathrm{GSR}}=3365 \mathrm{~km} \mathrm{~s}^{-1}$, and leads to a distance of $45 \mathrm{Mpc}\left(H_{0}=75 \mathrm{~km} \mathrm{~s}^{-1} \mathrm{Mpc}^{-1}\right)$ and to a scale of $217 \mathrm{pc}\left({ }^{\prime \prime}\right)^{-1}$. The velocity distribution along the line that joins both nuclei (Fig. 5) presents strong distortions with a steep gradient of $44 \mathrm{~km} \mathrm{~s}^{-1} \mathrm{Mpc}^{-1}$ along the bridge or tidal arm. The distributions of $\mathrm{H} \alpha$ flux (continuum subtracted) and the continuum (the signal at $60^{\prime \prime}$ corresponds to a star) are also shown in this figure. The $\mathrm{H} \alpha$ flux indicates that the star formation in this arm is comparable to that of the SE nucleus, and significantly lower than for the NW one. The continuum shows, besides the localized stellar contribution with about the same brightness in both nuclei, that the NW component presents bulge and a well marked disk, while the SE only has a large bulge, indicative of a rather early morphological type, reinforcing the morphological classification assigned earlier. This trace of disk in the NW component is compatible with the vestige of disk detected in the $J$ and $K$ images of the NASA/IPAC Infrared Science Archive. It is also compatible with the elongated shape that the nuclear region of this component presents in contraposition to the very regular and spherical shape of the SE component in R and IR images of the DSS. The Na I $\lambda 5893$ absorption line was detected in the continua emission of both nuclei (see Figs. 6a and b), and their corresponding stellar radial velocity dispersions resulted in $\sigma_{\mathrm{NW}}=(207 \pm 27) \mathrm{km} \mathrm{s}^{-1}$ and $\sigma_{\mathrm{SE}}=(147 \pm 16) \mathrm{km} \mathrm{s}^{-1}$. As a

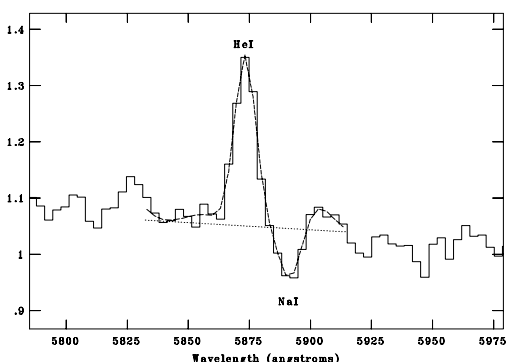

(a)

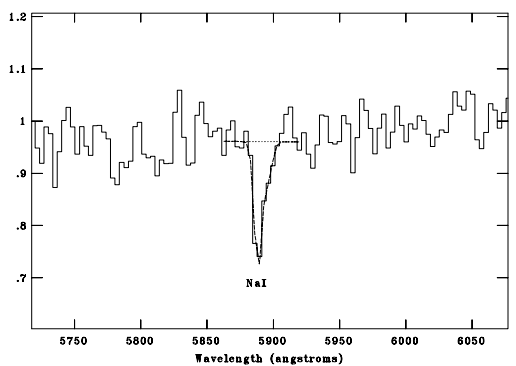

(b)

Fig. 6. a) Gaussian fitting of the $\mathrm{NaI} \lambda 5893$ absorption line in the spectrum of the NW nucleus. Note the adjacent nebular emission line He I $\lambda$ 5876. Ordinate in arbitrary units. b) Gaussian fitting of the $\mathrm{Na} I \lambda 5893$ absorption line in the spectrum of the SE nucleus. Ordinate in arbitrary units.

first approximation, the velocity dispersion of the bulges is still related with the original masses of the colliding system. This kind of approach has been used in previous works on merging systems where the central region is still not dynamically relaxed (e.g. Agüero et al. 2001), so from the obtained velocity dispersions and assuming a Maxwellian distribution it was possible to obtain the masses of the progenitor systems, which resulted in $M_{\mathrm{NW}} \approx 1.5 \times 10^{11} M_{\odot}$ and $M_{\mathrm{SE}} \approx 6 \times 10^{10} M_{\odot}$. When (i), considering an inclination angle of about $60^{\circ}$ for the NW component and about $20^{\circ}$ for the SE one; (ii) using the kinematical half width of the integrated $\mathrm{H} \alpha$ profile as an approximation for the amplitude of the rotation curve; and (iii) assuming that this curve remains flat up to the most external radius, the Keplerian mass resulted $(2.5 \pm 1) \times 10^{11} M_{\odot}$ for the NW component and $(5.2 \pm 2) \times 10^{10} M_{\odot}$ for the SE component. These values are similar to those obtained above. On the other hand, when adopting the ratio for moderate starbursts $L_{\mathrm{IR}} / M_{\mathrm{gas}}=20$ and assuming that about the $70 \%$ of $L_{\mathrm{IR}}$ also comes from the NW nucleus, indicative gas masses of the two components were derived: $M_{\mathrm{gasNW}} \approx 9 \times 10^{8} M_{\odot}$ and $M_{\mathrm{gasSE}} \approx 4 \times 10^{8} M_{\odot}$. Radial velocities were derived for the PA $=78^{\circ}$ (Fig. 7), which crosses the NW nucleus and the northern arm. The velocity of the arm is blueshifted compared to the velocity of the NW component, which is compatible with the numerical simulations, which are described in the next section. The radial velocity distribution is nearly flat for the points that belong to the NW component, so this position angle would correspond to the kinematic minor axis of this subsystem.

The derived abundances, burst ages (i.e. the time delay between bursts in both components of $\sim 1.6 \times 10^{7} \mathrm{yr}$ ), and masses suggest that the starburst in the SE component, the minor one, started earlier than the one in the NW component, in agreement with Bernlöhr (1993), and in a medium poorer in weighted elements. 
$\mathrm{R}(\mathrm{kpc})$

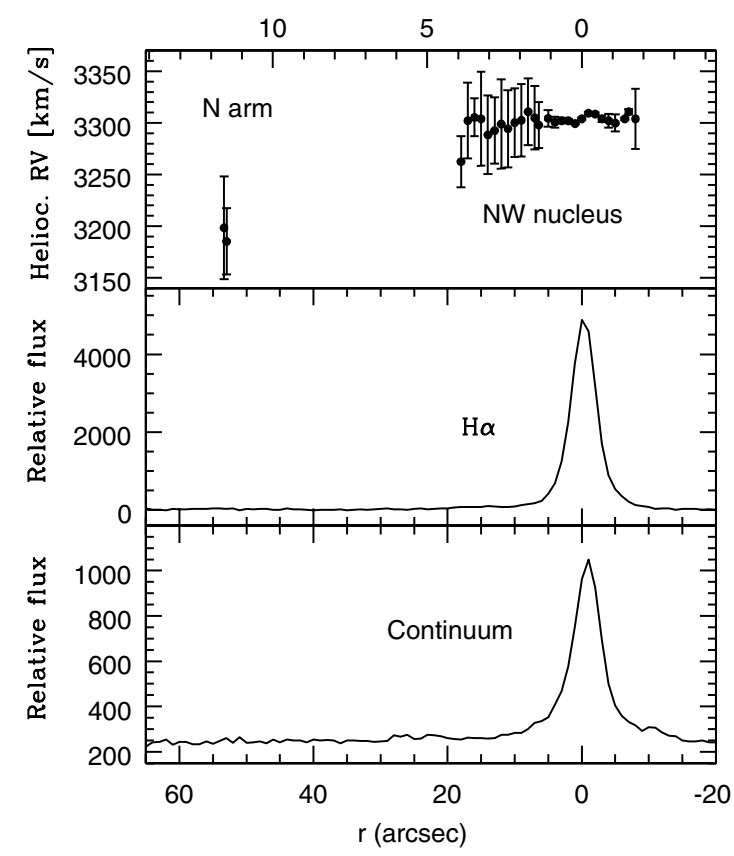

Fig. 7. (Top) Heliocentric radial velocity distribution along $\mathrm{PA}=78^{\circ}$. The velocity values were obtained from the Gaussian fitting of the $\mathrm{H} \alpha$ emission line and the radial velocity error was derived using the expression for $\sigma$ in Keel (1996), and the uncertainties shown in the figure are of $2 \sigma$. The origin is placed in the centre of the NW component and is considered positive in the direction of the northern arm. (Middle) $\mathrm{H} \alpha$ flux distribution (continuum subtracted) along the same position angle. (Bottom) Continuum distribution along the same position angle.

\subsection{Encounter modelling}

In order to understand the dynamical evolution of AM 1003-435, we simulated the encounter between the system components, following the evolution of their stellar and gaseous contents. Simulations were produced using GADGET-2 code (Springel et al. 2001; Springel 2005).

Galaxy models were constructed following the general prescription of Hernquist (1993), including a gas disk component. The initial models of each galaxy of the pair were constructed based on the observed sizes, masses, and radial velocity distributions. The NW component model was assumed to have a rotation curve that peaks at $180 \mathrm{~km} \mathrm{~s}^{-1}$ at $7 \mathrm{kpc}$, with a total mass of $1.5 \times 10^{11} M_{\odot}$ and made up of a stellar disk, a gas disk, a bulge, and dark-matter halo components. A total number of 25600 particles were used. For the SE component model a rotation curve was assumed that peaks at $130 \mathrm{~km} \mathrm{~s}^{-1}$ at $6 \mathrm{kpc}$, along with a total mass of $6 \times 10^{10} M_{\odot}$ and a similar set of components, but this time with a more prominent bulge. A total of 25600 particles were also used here. The dark-matter halos of both model galaxies have been assumed to have $\sim 3$ times the mass of the "visible" components (stellar and gaseous disk + bulge). This value is not well constrained by the observations but may be justified by the presence of well-developed tidal arms, indicative of a potential well that is not very deep and hence of a low-mass dark halo.The main parameters that describe the selected encounter model are listed in Table 3.

Assuming that there was only one close encounter by then, we searched for parabolic orbits that fullfilled the observational constraints, namely: the present position of the galaxies, the radial component of the velocity vectors of the galaxies, and the fact that the observed tidal arms are typical of a prograde encounter. This way, the free parameters were the pericentre distance, the line-of-sight component of the distance between the galaxies, and the radial velocity (within the range of the observational uncertainty). Out of this process we obtained a large amount of possible orbits, that we classify in families. A set of 15 simulations were performed, and the final choice was based on comparing the resulting morphologies and velocity distributions with the observational information.

Figure 8 shows the evolution of the encounter of the model that best reproduces the observational constraints, in which the galaxies approach each other along a parabolic orbit with a perigalactic distance $q=7.0 \mathrm{kpc}$. Time is shown in Myr in the upper right corner of each frame, with respect to the orbital pericentre. The frames that have been identified as the most similar to the observed system stage, given its morphology and kinematics, indicate that the perigalacticum would have occurred about $26 \mathrm{Myr}$ ago, which is quite comparable to the burst age derived for the SE component (20 Myr).

Star formation in both galaxies would have begun about the same time as or after the perigalacticum. The north-eastern tail would belong to the SE progenitor and would be bluer (see colour map from Reduzzi \& Rampazzo (1996); the orientation East-West is inverted in their description) than the western one, due to high content of gas and disk stars coming from the less massive and shattered SE body. According to the simulations, the bridge between the nuclei arises as a tidal tail with a high gas content, which would feed the observed star formation. As the bridge has the same observed colours as the NE tail, it seems plausible that this tail is also the place of star formation, in this case fed by the disk material of the SE progenitor.In the described scenario the galaxies will finally merge in about 1 Gyr.

\section{Conclusions}

The system AM 1003-435, composed of two strong interacting galaxies, was studied spectroscopically here. From the derived data the principal results are:

- The spectrum of the NW nucleus is typical of a starburst, while that of the SE one shows weak emission lines; their dominant stellar populations would be of $\mathrm{A}$ and $\mathrm{K}$ types respectively.

- The radial distributions of the relative oxygen and nitrogen abundances present the highest values at the NW nucleus, where they are comparable to those of galactic emission regions, since decreasing with the distance to it, as in many other spiral galaxies. The obtained electron temperatures and densities are in the range of normal values for $\mathrm{H}$ II regions.

- The equivalent widths $E W(\mathrm{H} \alpha)=119 \AA$ and $E W(\mathrm{H} \alpha+$ $[\mathrm{NII}])=173 \AA$ of the NW nucleus are indicative of very intense star formation activity confirming its starburst nature, while $E W(\mathrm{H} \alpha)=2 \AA$ was found for the SE nucleus. Indicative ages of $\sim 6.2 \times 10^{6} \mathrm{yr}$ and $\sim 2.2 \times 10^{7} \mathrm{yr}$, respectively were obtained for their bursts of star formation. The integrated spectrum of the NW component, typical of a starburst with $E W(\mathrm{H} \alpha)=87 \AA$ and $E W(\mathrm{H} \alpha+[\mathrm{N} \mathrm{II}])=126 \AA$ corresponds to a galaxy of $\mathrm{Sc} / \mathrm{Sm}$ type. The integrated spectrum of the SE component, characteristic of slightly active star formation objects, with $E W(\mathrm{H} \alpha) \sim 2 \AA$ corresponds to Sa-Sab galaxies. On the other hand, the values of $E W(\mathrm{H} \alpha)=46 \AA$ and $E W(\mathrm{H} \alpha+[\mathrm{N} \mathrm{III}])=62 \AA$ from the integrated spectrum of AM 1003-435 reflect the merger nature of the system, like its 
Table 3. Parameters used for the best model.

\begin{tabular}{|c|c|c|}
\hline & Model units* & Phys. units \\
\hline \multicolumn{3}{|l|}{ NW model parameters } \\
\hline Number of particles in stellar disk & 8192 & \\
\hline Disk total mass & 0.54 & $3.02 \times 10^{10} M_{\odot}$ \\
\hline Disk radial scale length & 0.7 & $2.45 \mathrm{kpc}$ \\
\hline Disk vertical scale thickness & 0.07 & $0.245 \mathrm{kpc}$ \\
\hline Reference radius $R_{\text {ref }}$ & 1.3 & $4.55 \mathrm{kpc}$ \\
\hline Toomre Q at $R_{\text {ref }}$ & 1.4 & \\
\hline Number of particles in gas disk & 8192 & \\
\hline Gas disk total mass & 0.054 & $3.02 \times 10^{9} M_{\odot}$ \\
\hline Gas disk radial scale length & 1.05 & $3.675 \mathrm{kpc}$ \\
\hline Gas disk vertical scale thickness & 0.021 & $0.0735 \mathrm{kpc}$ \\
\hline Toomre Q at $R_{\text {ref }}$ & 1.1 & \\
\hline Number of particles in bulge & 1024 & \\
\hline Bulge total mass & 0.054 & $3.02 \times 10^{9} M_{\odot}$ \\
\hline Bulge radial scale length & 0.14 & $0.49 \mathrm{kpc}$ \\
\hline Number of particles in halo & 8192 & \\
\hline Halo mass & 2.04 & $1.14 \times 10^{11} M_{\odot}$ \\
\hline Halo cutoff radius & 7.0 & $24.5 \mathrm{kpc}$ \\
\hline Halo core radius & 0.7 & $2.45 \mathrm{kpc}$ \\
\hline \multicolumn{3}{|l|}{ SE model parameters } \\
\hline Number of particles in stellar disk & 8192 & \\
\hline Disk total mass & 0.23 & $1.28 \times 10^{10} M_{\odot}$ \\
\hline Disk radial scale length & 0.6 & $2.1 \mathrm{kpc}$ \\
\hline Disk vertical scale thickness & 0.06 & $0.21 \mathrm{kpc}$ \\
\hline Reference radius $R_{\text {ref }}$ & 1.5 & $5.25 \mathrm{kpc}$ \\
\hline Toomre Q at $R_{\text {ref }}$ & 1.4 & \\
\hline Number of particles in gas disk & 8192 & \\
\hline Gas disk total mass & 0.023 & $1.28 \times 10^{9} M_{\odot}$ \\
\hline Gas disk radial scale length & 0.9 & $3.15 \mathrm{kpc}$ \\
\hline Gas disk vertical scale thickness & 0.004 & $0.014 \mathrm{kpc}$ \\
\hline Toomre Q at $R_{\text {ref }}$ & 1.1 & \\
\hline Number of particles in bulge & 1024 & \\
\hline Bulge total mass & 0.046 & $2.57 \times 10^{9} M_{\odot}$ \\
\hline Bulge radialscale length & 0.12 & $0.42 \mathrm{kpc}$ \\
\hline Number of particles in halo & 8192 & \\
\hline Halo mass & 0.86 & $4.80 \times 10^{10} M_{\odot}$ \\
\hline Halo cutoff radius & 5.7 & $19.95 \mathrm{kpc}$ \\
\hline Halo core radius & 0.57 & $1.995 \mathrm{kpc}$ \\
\hline \multicolumn{3}{|c|}{ Encounter parameters from our best model } \\
\hline Pericenter distance & 2.0 & $7.0 \mathrm{kpc}$ \\
\hline Present distance & 2.4 & $8.4 \mathrm{kpc}$ \\
\hline Pericenter vector direction ${ }^{* *}$ & $(-0.933,0.357,0.0473)$ & \\
\hline Orbital plane normal vector direction ${ }^{* * *}$ & $(0.067,0.0432,0.997)$ & \\
\hline Initial galaxy separation & 12.6 & $44.1 \mathrm{kpc}$ \\
\hline
\end{tabular}

Notes: * Simulations were done in a system of units with $G=1$. Model units scale to physical ones such that length unit is $3.5 \mathrm{kpc}$, unit velocity $262 \mathrm{~km} \mathrm{~s}^{-1}$, unit mass $5.586 \times 10^{10} M_{\odot}$, and unit time $13.062 \mathrm{Myr}{ }^{* *}$ Vector components defined in a Cartesian reference frame $(X, Y, Z)$, with $X$ to the North, $Y$ to the West, and $Z$ in the line of sight direction, with positive values towards the observer. ${ }^{* * *}$ The orbital plane's normal vector is the direction of the orbital angular momentum (reference frame defined as in ${ }^{*}$ ).

morphology suggests. Both morphological types derived here from the spectral characteristics agree with previous determinations based on photometric parameters. Different equivalent widths derived from the three mentioned integrated spectra would reflect that there is no global star-formation activity, and that this one is particularly intensified in the NW nuclear region. The $E W(\mathrm{H} \alpha)$ distribution shows that the starburst activity is appreciable in the apparent bridge and comparatively very low in the SE nuclear region, in spite of the supposedly present interactions. This suggests that AM 1003-435 is seen after the peak of activity of the SE progenitor.

- The H $\alpha$ luminosities derived from the integrated spectra of AM 1003-435 system and the NW component would be $\left(L_{\mathrm{H}_{\alpha}}\right)_{\mathrm{S}}=3 \times 10^{7} L_{\odot}$ and $\left(L_{\mathrm{H}_{\alpha}}\right)_{\mathrm{NW}}=2 \times 10^{7} L_{\odot}$ respectively, so about $70 \%$ of the $\left(L_{\mathrm{H}_{\alpha}}\right)_{\mathrm{S}}$ would correspond to the NW's component contribution.

The resulting infrared luminosity of the system $L_{\mathrm{IR}}=2.5 \times$ $10^{10} L_{\odot}$ is not high in relation to other mergers, but it is comparable to those of the archetypical starburst galaxies of moderate activity M 82, NGC 253, and NGC 7714. If the IR radiation has the same origin as the $\mathrm{H} \alpha$ emission, it would arise mostly from the NW component. The far infrared colour indexes $\alpha(60,25)$ and $\alpha(100,60)$ also point to AM 1003435 as a starburst, in agreement with the spectroscopic data.

The estimated star-formation rate for AM 1003-435 indicates that its activity is moderate, as it is very similar to those of M 82 and NGC 253. 


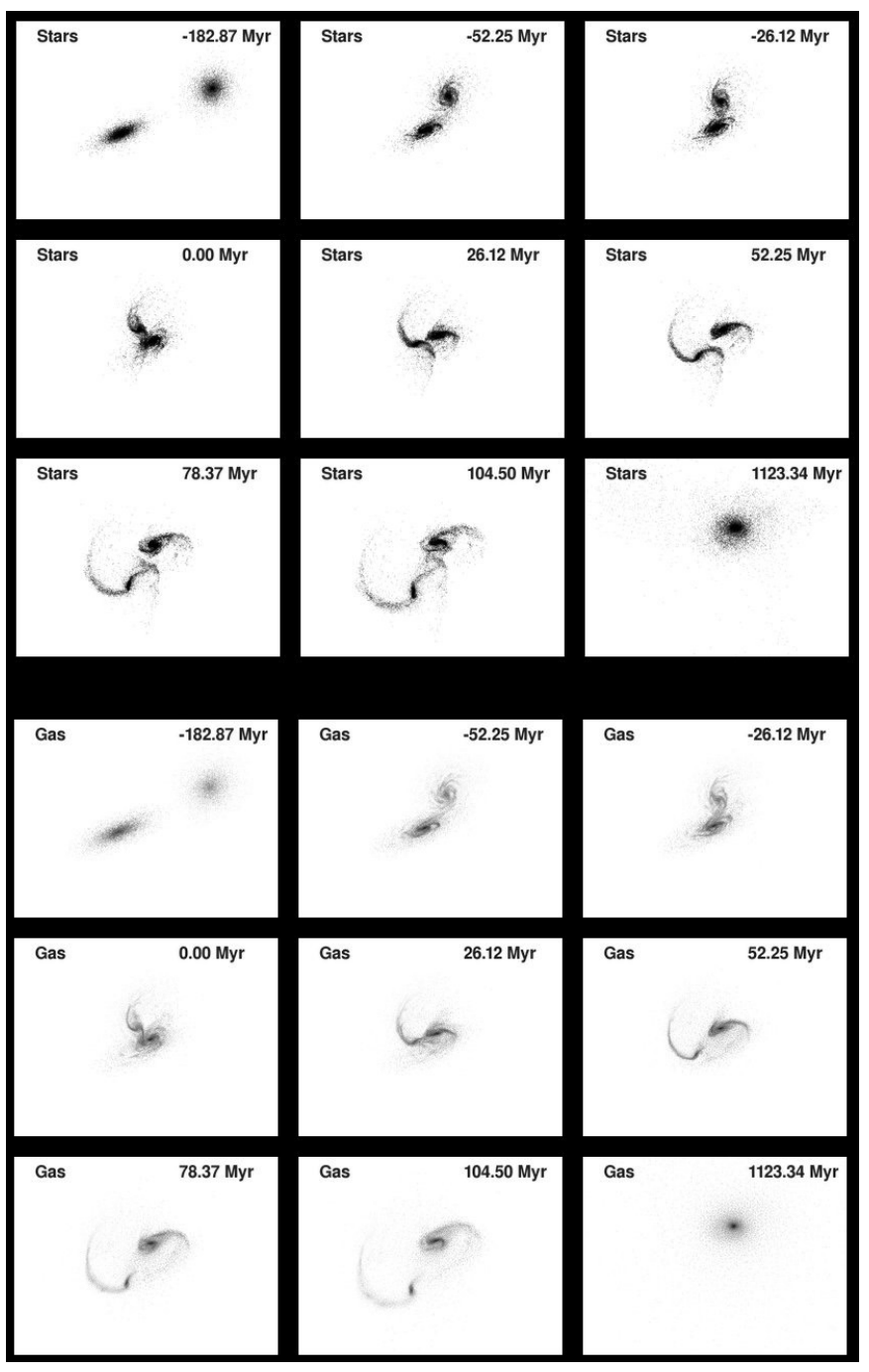

The $\mathrm{H} \alpha$ flux distribution reflects that the star formation in the bridge or tidal arm is comparable to the star-formation of the SE nucleus, and significantly lower than that of the NW one. The continuum flux distribution shows that the NW component presents both a bulge and a disk while the SE component only an important bulge, indicative of its rather early morphological type.

- The stellar radial velocity dispersions in both nuclei, derived from the $\mathrm{Na} I \lambda 5893$ absorption line, resulted in $\sigma_{\mathrm{NW}}=(207 \pm$ 27) $\mathrm{km} \mathrm{s}^{-1}$ and $\sigma_{\mathrm{SE}}=(147 \pm 16) \mathrm{km} \mathrm{s}^{-1}$, and the masses of their respective progenitor systems are $M_{\mathrm{NW}} \approx 1.5 \times 10^{11} M_{\odot}$ and $M_{\mathrm{SE}} \approx 6 \times 10^{10} M_{\odot}$. The resulting abundances, burst ages, and masses suggest that the starburst in the SE component, the minor one, started earlier, in a medium poorer in weighted elements than the NW medium.

- On the other hand, from a set of $N$-body simulations of the encounter between both components using GADGET-2 $(2001,2005)$ code, it was found that the perigalacticum would have occurred about $26 \mathrm{Myr}$ ago, which is comparable to the burst age derived for the SE component. Star formation in both galaxies would have begun after the perigalacticum. The eastern tail would belong to the SE progenitor and would be bluer due to high content of disk stars of the less massive and shattered SE body. The bridge, with the same colours as the NE tail, is also a tidal feature with star formation, as is shown by the spectroscopy. In this scenario the central bodies will merge in about 1 Gyr.
Fig. 8. Evolution of the selected encounter model (Table 3), the stellar component is depicted in the nine upper panels and the gaseous component in the lower panels. Time is shown in Myr in the upper right corner of each frame, with respect to the orbital pericenter. The observed system stage would correspond to the +26.12 Myr frames.

\section{References}

Agüero, E. L., Paolantonio, S., \& Günthardt, G. 2000, AJ, 119, 94 Agüero, E. L., Díaz, R. J., \& Paolantonio, S. 2001, PASP, 113, 1515, Bernlöhr, K. 1993, A\&A, 268, 25

Bica, E. 1988, A\&A, 195, 76

Díaz, A. I. 1985, Ph.D. Thesis, Univ. of Sussex

Edmunds, M. J., \& Pagel, B. E. J. 1984, MNRAS, 211, 507

Evans, I. 1986, ApJ 309, 544

Hernquist, L. 1993, ApJS, 86, 389

Joseph, R. D., Meikle, W.P. S., Robertson, N. A., \& Wright, G. S. 1984, MNRAS, 209, 111

Keel, W. C. 1996, ApJS, 106, 27

Kennicutt, R. C. Jr. 1992, ApJS, 79, 255

Lauberts, A., \& Valentijn, E. 1989, The Surface Photometry Catalog of the ESOUppsala Galaxies, ESO, Garching bei München

Leitherer, C., Schaerer, D., Goldader, J. D., et al. 1999, ApJS, 123, 3

Liu, C. T., \& Kennicutt, R. C. Jr. 1995, ApJ, 450, 547

McCall, M. L., Rybski, P. M., \& Shields, G. A. 1985, ApJS, 57, 1

Moorwood, A. F. M. 1996, SSRv, 77, 303

Osterbrock, D. E. 1989, Astrophysics of Gaseous Nebulae and Active Galactic

Nuclei (Mill Valley: University Science Books)

Reduzzi, L., \& Rampazzo, R. 1996, A\&A, 116, 515

Sanders, D. B., \& Mirabel, I. F. 1996, ARA\&A, 34, 749

Scoville, N., \& Young, J. S. 1983, ApJ, 265, 148

Seaton, M. J. 1979, MNRAS, 187, 73P

Sekiguchi, K., \& Wostencroft, R. D. 1992, MNRAS, 255, 581

Shields, G. 1990, ARA\&A, 28, 525

Springel, V. 2005, MNRAS, submitted

Springel V., Yoshida N., \& White S. D. M. 2001, New Astronomy, 6, 51

Stone, R. P. S., \& Baldwin, J. A. 1982, MNRAS, 204, 347

Veilleux, S., \& Osterbrock, D. E. 1987, ApJS, 63, 295 
G. I. Günthardt et al.: AM 1003-435, Online Material p 1

\section{Online Material}


Table 1. Line intensities relative to $\mathrm{H} \beta$.

\begin{tabular}{|c|c|c|c|c|c|c|c|c|c|c|c|c|c|}
\hline \multicolumn{14}{|c|}{$\begin{array}{c}F_{\lambda} / F_{\beta} \\
I_{\lambda} / I_{\beta}\end{array}$} \\
\hline Line & $\begin{array}{c}\lambda \\
(\AA)\end{array}$ & $\begin{array}{c}\text { Reg. } 1 \\
40.9\end{array}$ & $\begin{array}{c}\text { Reg. } 2 \\
36 . .4\end{array}$ & $\begin{array}{c}\text { Reg. } 3 \\
344^{\prime \prime} 8\end{array}$ & $\begin{array}{l}\text { Reg. } 4 \\
24 . .4\end{array}$ & $\begin{array}{c}\text { Reg. } 5 \\
16 .{ }^{\prime \prime} 8\end{array}$ & $\begin{array}{c}\text { Reg. } 6 \\
10 ! 7\end{array}$ & $\begin{array}{c}\text { Reg. } 7 \\
6 ! 1\end{array}$ & $\begin{array}{c}\text { Reg. } 8 \\
3{ }^{\prime \prime} 1\end{array}$ & $\begin{array}{c}\text { Reg. } 9 \\
0^{\prime \prime}\end{array}$ & $\begin{array}{c}\text { Reg. } 10 \\
-3{ }^{\prime \prime} 1\end{array}$ & $\begin{array}{c}\text { Reg. } 11 \\
-6 .{ }^{\prime \prime} 1\end{array}$ & $\begin{array}{c}\text { Reg. 12 } \\
-9 ! .1\end{array}$ \\
\hline \multirow[t]{2}{*}{$\mathrm{H} \beta$} & 4861 & $1.00 \mathrm{c}$ & $1.00 \mathrm{c}$ & $1.00 \mathrm{c}$ & $1.00 \mathrm{c}$ & $1.00 \mathrm{c}$ & $1.00 \mathrm{c}$ & $1.00 \mathrm{a}$ & $1.00 \mathrm{~b}$ & $1.00 \mathrm{a}$ & $1.00 \mathrm{a}$ & $1.00 \mathrm{~b}$ & $1.00 \mathrm{c}$ \\
\hline & & 1.00 & 1.00 & 1.00 & 1.00 & 1.00 & 1.00 & 1.00 & 1.00 & 1.00 & 1.00 & 1.00 & 1.00 \\
\hline \multirow[t]{2}{*}{ [O III] } & 4959 & - & $1.16 \mathrm{c}$ & - & $1.32 \mathrm{c}$ & $0.74 \mathrm{c}$ & - & $0.97 \mathrm{c}$ & $0.34 \mathrm{a}$ & $0.31 \mathrm{a}$ & $0.28 b$ & $0.60 \mathrm{c}$ & $1.23 \mathrm{c}$ \\
\hline & & - & 1.13 & - & 1.29 & 0.70 & - & 0.95 & 0.33 & 0.30 & 0.27 & 0.58 & 1.14 \\
\hline \multirow[t]{2}{*}{ [O III] } & 5007 & $4.46 c$ & $3.45 \mathrm{c}$ & - & $3.21 \mathrm{c}$ & $2.56 \mathrm{c}$ & - & $1.52 \mathrm{c}$ & $0.96 a$ & $0.86 a$ & $0.90 \mathrm{a}$ & $1.55 \mathrm{~b}$ & $3.05 \mathrm{c}$ \\
\hline & & 4.04 & 3.35 & - & 3.15 & 2.36 & - & 1.46 & 0.93 & 0.83 & 0.87 & 1.49 & 2.76 \\
\hline \multirow[t]{2}{*}{ [N II] } & 6548 & $0.99 \mathrm{c}$ & - & - & - & - & - & $0.60 \mathrm{c}$ & $0.47 \mathrm{~b}$ & $0.60 \mathrm{a}$ & $0.42 \mathrm{a}$ & $0.37 \mathrm{c}$ & - \\
\hline & & 0.39 & - & - & - & - & - & 0.42 & 0.35 & 0.43 & 0.30 & 0.26 & - \\
\hline \multirow[t]{2}{*}{$\mathrm{H} \alpha$} & 6563 & $7.20 \mathrm{c}$ & $3.80 \mathrm{c}$ & $3.28 \mathrm{c}$ & $3.41 \mathrm{c}$ & $6.06 \mathrm{c}$ & $7.12 \mathrm{c}$ & $4.02 \mathrm{~b}$ & $3.80 \mathrm{a}$ & $3.96 \mathrm{a}$ & $3.97 \mathrm{a}$ & $4.16 \mathrm{~b}$ & $7.46 c$ \\
\hline & & 2.85 & 2.85 & 2.85 & 2.85 & 2.85 & 2.85 & 2.85 & 2.85 & 2.85 & 2.85 & 2.85 & 2.85 \\
\hline \multirow[t]{2}{*}{ [N II] } & 6583 & - & - & - & $1.04 \mathrm{c}$ & $1.21 \mathrm{c}$ & - & $1.07 \mathrm{c}$ & $1.24 \mathrm{a}$ & $1.22 \mathrm{a}$ & $1.24 \mathrm{a}$ & $1.19 b$ & $1.52 \mathrm{c}$ \\
\hline & & - & - & - & 0.86 & 0.57 & - & 0.76 & 0.93 & 0.87 & 0.89 & 0.81 & 0.57 \\
\hline \multirow[t]{2}{*}{ [S II] } & 6717 & - & - & - & - & - & - & - & $0.60 \mathrm{a}$ & $0.62 \mathrm{a}$ & $0.71 \mathrm{a}$ & $1.15 b$ & $1.59 \mathrm{c}$ \\
\hline & & - & - & - & - & - & - & - & 0.44 & 0.44 & 0.50 & 0.77 & 0.57 \\
\hline \multirow[t]{2}{*}{ [S II] } & 6731 & - & - & - & - & - & - & - & $0.38 b$ & $1.38 \mathrm{a}$ & $0.48 \mathrm{a}$ & $0.68 b$ & - \\
\hline & & - & - & - & - & - & - & - & 0.28 & 0.27 & 0.33 & 0.45 & - \\
\hline$C$ & & 1.04 & 0.17 & - & 0.03 & 0.80 & - & 0.25 & 0.17 & 0.22 & 0.23 & 0.29 & 1.08 \\
\hline $\log I \beta$ & & -14.55 & -14.98 & - & -14.63 & -14.22 & - & -14.80 & -13.88 & -13.48 & -13.78 & -14.43 & -14.05 \\
\hline
\end{tabular}

Note: $F_{\lambda}$ is the observed flux. $I_{\lambda}$ is the reddening corrected flux in units of $\mathrm{erg} \mathrm{cm} \mathrm{cm}^{-2} \mathrm{~s}^{-1} C$ is the $\mathrm{H} \beta$ logarithmic extintion.

Estimated errors: $\mathrm{a} \leq 10 \%, 10 \% \leq \mathrm{b} \leq 20 \%, 20 \% \leq \mathrm{c}$. Distances are given with respect to region 9, being positives towards the Southeast. 